\title{
Brown dwarfs in the Pleiades cluster
}

\author{
III. A deep $I Z$ survey $^{\star, \star \star}$
}

\author{
M.R. Zapatero Osorio ${ }^{1}$, R. Rebolo ${ }^{1}$, E.L. Martín ${ }^{1, \star \star \star}$, S.T. Hodgkin ${ }^{2}$, M.R. Cossburn ${ }^{2}$, \\ A. Magazzù ${ }^{3, \star \star \star \star}$, I.A. Steele ${ }^{4}$, and R.F. Jameson ${ }^{2}$ \\ 1 Instituto de Astrofísica de Canarias, C/, Vía Láctea s/n, E-38200 La Laguna, Tenerife, Spain \\ 2 Astronomy Group, Department of Physics and Astronomy, University of Leicester, Leicester LE1 7RH, UK \\ 3 Centro Galileo Galilei, Apartado 565, E-38700 Santa Cruz de La Palma, Islas Canarias, Spain \\ 4 Astrophysics Research Institute, Liverpool John Moores University, Liverpool L3 3AF, UK
}

Received August 5; accepted September 16, 1998

\begin{abstract}
We present the results of a deep CCD-based $I Z$ photometric survey of a $\sim 1 \mathrm{deg}^{2}$ area in the central region of the Pleiades Galactic open cluster. The magnitude coverage of our survey (from $I \sim 17.5$ down to 22 ) allows us to detect substellar candidates with masses between 0.075 and $0.03 M_{\odot}$. Details of the photometric reduction and selection criteria are given. Finder charts prepared from the $I$-band images are provided.
\end{abstract}

Key words: stars: low-mass, brown dwarfs - Galaxy: open clusters and associations: Pleiades catalogs

\section{Introduction}

The Pleiades star cluster is an ideal hunting ground for substellar objects mainly due to its richness of members, young age, proximity and scarce interstellar absorption. Taking advantage of these properties, several photometric searches aimed at finding brown dwarfs (BDs) have been performed during the last decade (see Hambly 1998

Send offprint requests to: M.R. Zapatero Osorio

* Based on observations made with the Isaac Newton Telescope (INT) and the Jacobus Kaptein Telescope (JKT) operated on the island of La Palma by the Isaac Newton Group at the Observatorio del Roque de los Muchachos of the Instituto de Astrofísica de Canarias; and on observations made with the Nordic Optical Telescope (NOT) at the same observatory.

$\star \star$ Figure 5 is only available at the

http://www.edpsciences.com

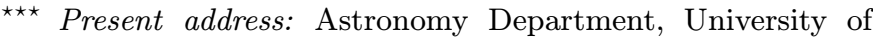
California, Berkeley, CA 94720, U.S.A.

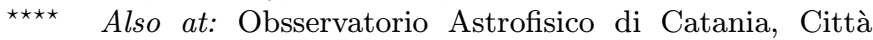
Universitaria, Viale Andrea Doria 6, I-95125 Catania, Italy.

Correspondence to: mosorio@ll.iac.es for a review). The recent spectroscopic confirmations of Pleiades objects at the stellar-substellar boundary and genuine substellar members (Basri et al. 1996; Rebolo et al. 1996; Martín et al. 1998; Stauffer et al. 1998) previously discovered as a result of optical photometric surveys in small areas suggest that a numerous population of very low-mass objects may be found in this cluster. This encourages future surveys to discover BDs cooler and less massive than those $\left(0.075-0.05 M_{\odot}\right)$ previously detected by these surveys. The Pleiades offers a unique opportunity to establish the observational properties of these rather elusive objects and to characterize the initial mass function in the substellar mass regime.

\subsection{The survey}

As part of an on-going search for BDs in the Pleiades, we have conducted a deep CCD-based $I Z$ survey using the $2.5 \mathrm{~m}$ Isaac Newton Telescope (INT) located on the Observatorio del Roque de los Muchachos (ORM, island of La Palma). The area covered was $1.05 \mathrm{deg}^{2}$ within the central region of the cluster (a small fraction of the total area was also observed using the $R$ filter). More than 40 faint $(I \geq 17.5)$, very red $(I-Z \geq 0.5)$ objects have been detected down to $I \sim 22$. Their location in the colourmagnitude diagram suggests cluster membership. In this paper we report on the details of this survey along with the selection criteria. We provide $I Z$ magnitudes, coordinates and finder charts for all candidates. Preliminary results of this survey were presented in Zapatero Osorio et al. (1997a, 1998a). An extensive discussion on the membership of the candidates and derivation of the initial mass function will be given in a forthcoming paper (Zapatero Osorio et al. 1998b). 
Table 1. Field $\left(100 \operatorname{arcmin}^{2}\right)$ center coordinates

\begin{tabular}{|c|c|c|c|c|}
\hline \multicolumn{2}{|c|}{$\begin{array}{l}\text { RA (J2000) DEC } \\
\left(\begin{array}{lll}\mathrm{h} & \mathrm{m} & \mathrm{s}\end{array}\right) \quad\left(\begin{array}{lll}0 & \prime & \prime \prime\end{array}\right)\end{array}$} & $\begin{array}{c}\text { Date } \\
(1996)\end{array}$ & $\begin{array}{c}\text { Sep. }^{\mathrm{a}} \\
(\text { arcmin })\end{array}$ & Filter \\
\hline 34335 & 243000 & 21 Sep. & 53.02 & $I Z$ \\
\hline 34343 & 244500 & $12 \mathrm{Feb}$. & 58.69 & $R I$ \\
\hline 34415 & 234030 & 21 Sep. & 46.15 & $I Z$ \\
\hline 34430 & 235500 & 21 Sep. & 36.32 & $I Z$ \\
\hline 34430 & 244000 & 20 Sep. & 47.44 & $I Z$ \\
\hline 34500 & 243700 & 20 Sep. & 40.54 & $I Z$ \\
\hline 34515 & 235435 & 20 Sep. & 27.02 & $I Z$ \\
\hline 34545 & 241000 & 20 Sep. & 17.37 & $I Z$ \\
\hline 34545 & 244600 & 20 Sep. & 42.55 & $I Z$ \\
\hline 34550 & 235500 & 20 Sep. & 20.00 & $I Z$ \\
\hline 34625 & 244200 & 20 Sep. & 35.89 & $I Z$ \\
\hline 34630 & 242500 & 20 Sep. & 19.25 & $I Z$ \\
\hline 34630 & 243700 & 21 Sep. & 30.77 & $I Z$ \\
\hline 34720 & 232500 & 21 Sep. & 42.25 & $I Z$ \\
\hline 34722 & 223940 & 13 Feb. & 87.48 & $R I Z$ \\
\hline 34730 & 242600 & 20 Sep. & 20.19 & $I Z$ \\
\hline 34752 & 241000 & 21 Sep. & 12.23 & $I Z$ \\
\hline 34800 & 240000 & 21 Sep. & 15.39 & $I Z$ \\
\hline 34800 & 243400 & 9 Feb. & 30.25 & $R I Z$ \\
\hline 34805 & 233932 & 9 Feb. & 31.24 & $R I Z$ \\
\hline 34805 & 244500 & 20 Sep. & 40.76 & $I Z$ \\
\hline $348 \quad 15$ & 233230 & 21 Sep. & 38.55 & $I Z$ \\
\hline 34815 & 242800 & 21 Sep. & 27.06 & $I Z$ \\
\hline $\begin{array}{lll}3 & 48 & 19\end{array}$ & 253200 & 11 Feb. & 86.85 & $R I$ \\
\hline 34820 & 234500 & 20 Sep. & 28.62 & $I Z$ \\
\hline 34830 & 235700 & 21 Sep. & 22.87 & $I Z$ \\
\hline 34840 & 224200 & 13 Feb. & 88.07 & $R I Z$ \\
\hline 34840 & 244500 & 20 Sep., 13 Feb. & 44.27 & $I Z$ \\
\hline 34845 & 234000 & 20-21 Sep. & 36.15 & $I Z$ \\
\hline 34857 & 241800 & 21 Sep. & 28.84 & $I Z$ \\
\hline 34915 & 243100 & 20 Sep. & 38.97 & $I Z$ \\
\hline 34920 & 233200 & 21 Sep. & 47.48 & $I Z$ \\
\hline 34920 & 244500 & 20 Sep. & 49.54 & $I Z$ \\
\hline 34935 & 235500 & 21 Sep. & 37.40 & $I Z$ \\
\hline 34941 & 251540 & 11 Feb. & 77.72 & $R I$ \\
\hline 35000 & 242800 & 20 Sep. & 46.03 & $I Z$ \\
\hline 35015 & 234000 & 21 Sep. & 52.18 & $I Z$ \\
\hline 35025 & 240500 & 21 Sep. & 46.83 & $I Z$ \\
\hline 35030 & 235000 & 21 Sep. & 50.94 & $I Z$ \\
\hline 35330 & 234000 & $11 \mathrm{Feb}$. & 93.29 & $R I$ \\
\hline
\end{tabular}

a Separation from the cluster center $\left(3^{\mathrm{h}} 47^{\mathrm{m}},+24^{\circ} 7^{\prime}\right)$.

\section{Observations and data reduction}

All of our CCD survey was carried out during 1996 in two campaigns which took place on February 9-12 and on September 19-20. We used the TEK $\left(1024 \times 1024\right.$ pixel $\left.^{2}\right)$ detector mounted on the prime focus of the telescope, with a field of view of $10 \times 10 \mathrm{arcmin}^{2}$. A total of 40 fields, with center coordinates listed in Table 1 , were observed with the Harris $(R) I$ and RGO $Z$ broad-band filters providing a total survey area of $1.05 \mathrm{deg}^{2}(\sim 6.5 \%$ of the whole cluster). Most of these fields were selected to avoid very bright stars and were located within $1 \mathrm{deg}$ of the innermost region of the Pleiades (see Table 1), where the population of $\mathrm{M}$ dwarf proper motion members is much larger than in outer areas (Hambly et al. 1993; Bouvier et al. 1998). If as expected less massive Pleiades members show a similar

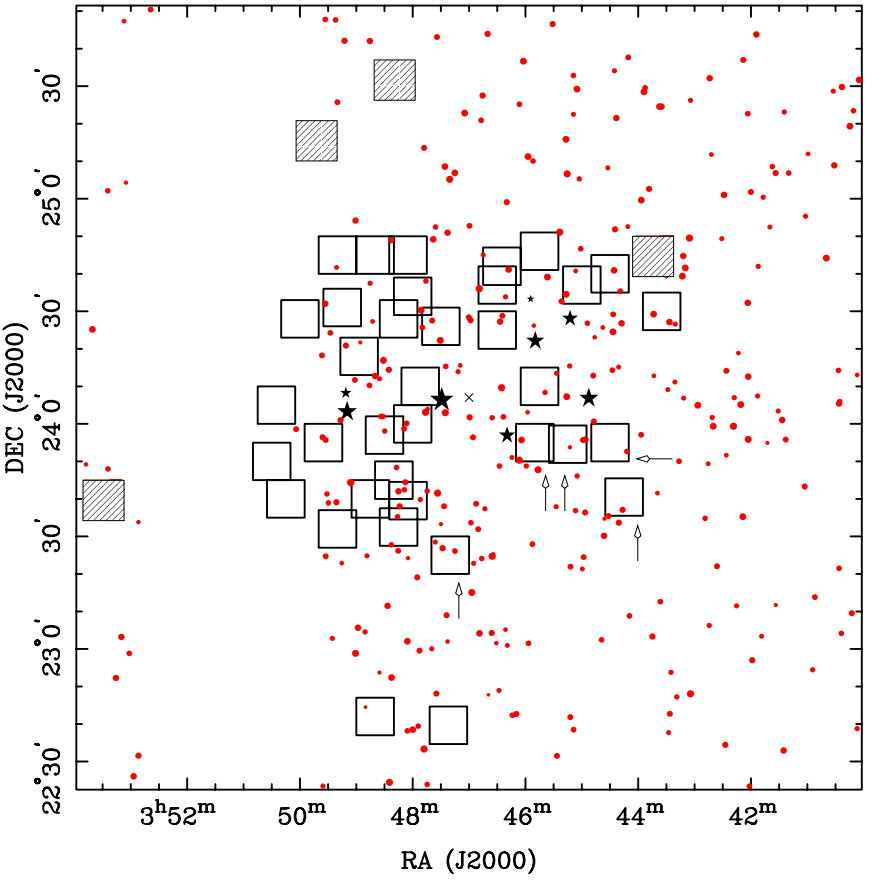

Fig. 1. Location of our fields (squares) within $3.5 \times 3.5$ of the Pleiades area. Open squares stand for those fields observed with $I Z$ filters while the shaded squares depict the four fields observed with $R I$ filters. Central coordinates (indicated with a cross) are $3^{\mathrm{h}} 47^{\mathrm{m}},+24^{\circ} 7^{\prime}$ (Eq. 2000). The five fields which may have some amount of extra reddening according to the $\mathrm{CO}$ contours shown by Breger (1987) are indicated with an arrow. Filled star symbols stand for stars brighter than $6^{\text {th }}$ magnitude, and filled circles for proper motion M members (Hambly et al. 1993) with $I$ magnitudes in the range 13-18. The vertical gap in the $\mathrm{M}$ star distribution around $3^{\mathrm{h}} 51^{\mathrm{m}}$ is due to the fact that there were no overlaps between the first and second epoch plates used by the authors, causing the lack of proper motion measurements for stars in that strip. The relative brightness is represented by symbol diameters. North is up and East is left

spatial distribution within the cluster our survey should be able to detect a large number of $\mathrm{BD}$ candidates. In Fig. 1 the location of all the frames obtained during the two observing runs is presented to scale. None of them (except for five fields indicated in the figure) falls within the small region southwest of the cluster center which is well known to suffer from high absorption (van Leeuwen 1983; Breger 1987; Stauffer \& Hartmann 1987). A small fraction $(10.5 \%)$ of our covered area was imaged in the three bands while a similar area was observed only in $R I$ filters. Weather conditions were always photometric, and the seeing oscillated between $1^{\prime \prime}$ and $1^{\prime \prime} .5$. Typical exposure times ranged from $10 \mathrm{~min}$ for the $R$ filter to $5 \mathrm{~min}$ for the $I$ and $Z$-bands.

We adopted the $I Z$ broad-band filters for several reasons. One of our goals was to detect objects fainter and less massive than the two cluster BDs Teide 1 and Calar 3 (M8, $I \sim 19, R-I \sim 2.6, \sim 0.055 M_{\odot}$, Rebolo et al. 1995; Martín et al. 1996). Theoretical evolutionary models 
(which do not include grain formation in very cool atmospheres) predict that these objects become much redder with colours $(R-I) \geq 3$ (Chabrier et al. 1996). Thus, they might be extremely faint in $R$ wavelengths, greatly hindering their detection. On the other hand, field stars do exhibit a turn-off in $(R-I)$ at around M7 spectral type, with stars of later types having bluer colours (Bessell 1991). The fluxes and colours of the Pleiades BDs fainter than Teide 1 and Calar 3 are unknown, but we expect them to have spectral energy distributions which resemble those of the coolest objects in the field. It could turn out that the $(R-I)$ colour is no longer useful to discriminate low luminosity cluster members from field objects. The $(I-J)$ colour, however, gets monotonically redder for lower temperatures (both for observed and theoretical predictions), implying that the slope of the spectral pseudocontinuum between $I$ and $J$ wavelengths clearly increases. As the $Z$ filter is centered at $920 \mathrm{~nm}$, we expect a similar behaviour with $I$ and $Z$. Although the efficiency of the CCD drops considerably in the $Z$-band, this effect is compensated by the increased brightness of BDs at these near-IR wavelengths. The $(I-Z)$ colour has been shown to be a useful discriminant for Pleiades BDs by Cossburn et al. (1997).

Other photometric searches for substellar objects in the Pleiades carried out with $R$ and $I$ (Jameson \& Skillen 1989; Zapatero Osorio et al. 1997b, Paper I) provide a high number of mid- and late-M stars that do not belong to the cluster and are contaminating the surveys. It is desiderable to find a strategy which avoids these field contaminants and facilitates a more efficient tool for detecting true members. In Paper I the success rate was only 25\%: two out of the eight proposed cool candidates have been confirmed as genuine Pleiades BDs (Rebolo et al. 1996). The authors argue that this was due to the detection of reddened late-M dwarfs (Zapatero Osorio et al. 1997c, Paper II). The use of longer wavelength filters would help to jump over this obstacle.

Raw frames were processed using standard techniques within the $\operatorname{IRAF}^{1}$ (Image Reduction and Analysis Facility) environment, which included bias subtraction, flat-fielding and correction for bad pixels by interpolation with values from the nearest-neighbour pixels. The photometric PSF fitting analysis was carried out using routines within DAOPHOT, which provides image profile information needed to discriminate between stars and galaxies. Instrumental $R I$ magnitudes were corrected for atmospheric extinction and transformed into the $R I$ Cousins system using observations of standard stars from Landolt's (1992) list. Special care was taken in including red standard stars in order to ensure a reliable transformation for the reddest candidates: the field SA 98 contains many pho-

\footnotetext{
${ }^{1}$ IRAF is distributed by National Optical Astronomy Observatories, which is operated by the Association of Universities for Research in Astronomy, Inc., under contract with the National Science Foundation.
}

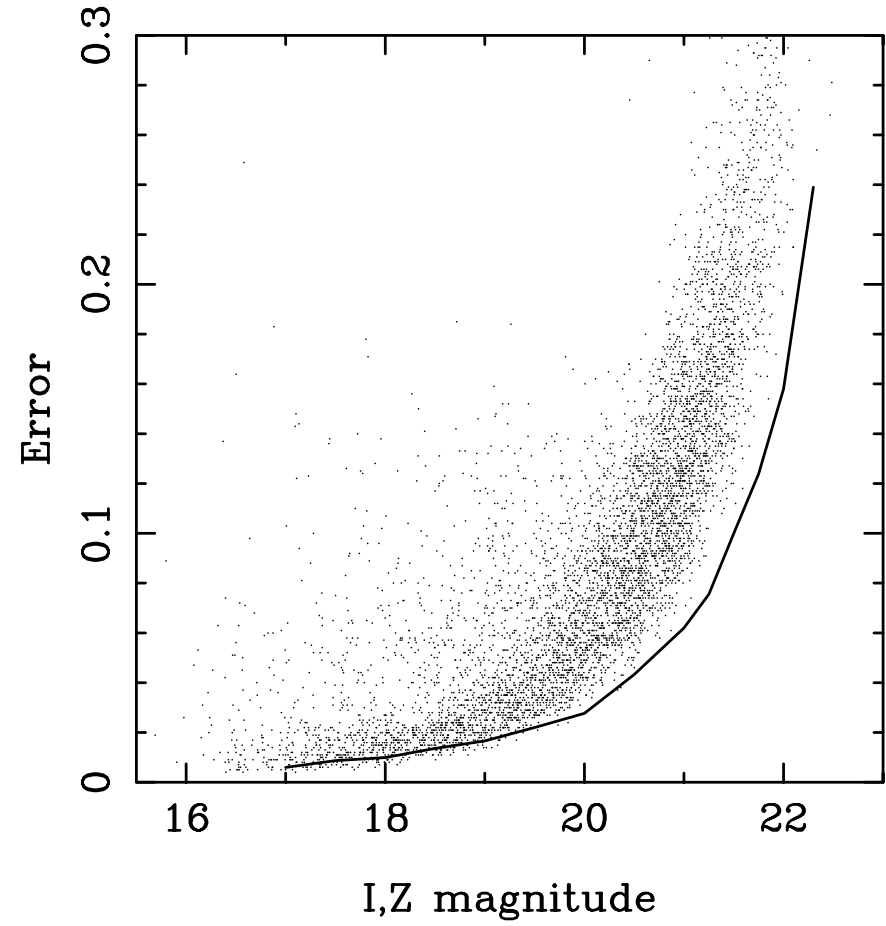

Fig. 2. Photometric errors as a function of observed $I$ (full line) and $Z$ (dots) magnitudes. The observed dispersion in the errors for the $I$-band is similar to that of the $Z$-band

tometric standards covering colours from A0 to M7 spectral type. The calibration of $Z$ magnitudes required more observational effort as there are no real data for standards available in the literature. We have not performed an absolute flux calibration for this filter, but obtained $(I-Z)$ colours with respect to a given spectral type. Using the same Landolt fields as observed through the other two filters at culmination (airmass $=1.1$ ), we set $Z=I$ for those standard stars with $(R-I) \sim 0$ (A0-type). The adopted $(I-Z)$ colours are shown in Table 2. Observations of these fields at different elevations allowed us to correct $Z$ instrumental magnitudes for atmospheric extinction. Errors for $Z$ instrumental magnitudes as provided by IRAF routines are plotted in Fig. 2. The best power law fit to the errors in $I$ for the bulk of data is superimposed in the figure for comparison. Summarizing, uncertainties in the INT photometry range from $\leq 0.05 \mathrm{mag}$ at $I, Z \sim 20.5,19.7$ to about $0.15 \mathrm{mag}$ at $22,21 \mathrm{mag}$, respectively.

We present in Fig. 3 the resulting $I$ vs. $(I-Z)$ diagram where data for the Pleiads HHJ $3, \mathrm{PPl} 15$ and Teide 1 (which are present in three of our fields) are combined with the new observations. We remark that $Z$ magnitudes are not on a standard system. Completeness and limiting magnitudes of our survey were derived following the same procedure described in Stauffer et al. (1994) and Paper I. We estimate them to be $I, Z \sim 21,20.5$ for completeness and 22.2, 21.5 for the limit. These values are indicated in the figure. Because there are almost no measurements in the $Z$-band of other cluster members, it is rather 
Table 2. Adopted $(I-Z)$ colours for the photometric standard stars used in the calibrations

\begin{tabular}{lcrc}
\hline SA Star & $(R-I)^{a}$ & $I^{a}$ & $(I-Z)^{b}$ \\
\hline 98642 & $0.393 \pm 0.002$ & $14.594 \pm 0.026$ & $0.15 \pm 0.09$ \\
98650 & $0.086 \pm 0.002$ & $12.105 \pm 0.003$ & $0.02 \pm 0.02$ \\
98652 & $0.339 \pm 0.024$ & $14.201 \pm 0.025$ & $0.07 \pm 0.04$ \\
98653 & $0.008 \pm 0.001$ & $9.522 \pm 0.002$ & $0.00 \pm 0.02$ \\
98670 & $0.653 \pm 0.001$ & $10.555 \pm 0.003$ & $0.23 \pm 0.03$ \\
98671 & $0.494 \pm 0.004$ & $12.315 \pm 0.006$ & $0.13 \pm 0.03$ \\
98675 & $1.002 \pm 0.002$ & $11.314 \pm 0.004$ & $0.39 \pm 0.03$ \\
98676 & $0.673 \pm 0.022$ & $11.716 \pm 0.005$ & $0.24 \pm 0.02$ \\
98682 & $0.352 \pm 0.003$ & $13.032 \pm 0.006$ & $0.26 \pm 0.05$ \\
98685 & $0.280 \pm 0.002$ & $11.384 \pm 0.005$ & $0.05 \pm 0.02$ \\
98 L5 & $2.60 \pm 0.04$ & $12.05 \pm 0.20$ & $1.1 \pm 0.2$ \\
\hline
\end{tabular}

a $R I$ magnitudes and their errors taken from Landolt (1992).

${ }^{b}$ 1- $\sigma$ errors come from uncertainties in $I$-band and the dispersion in the calibration.

difficult to establish the separation between Pleiads and field objects in our diagram. However, we have made an attempt to separate these two kinds of objects by plotting a straight line in Fig. 3 which is parallel to the photometric sequence defined by HHJ 3, PPl 15 and Teide 1 and shifted $0.3 \mathrm{mag}$ towards the blue. For bluer colours than those indicated by the line, the number of detections increases very drastically, probably indicating that these are field objects. On the other hand, the photometric dispersion observed in other optical and infrared colours among low-mass proper motion members is about $0.6-0.7 \mathrm{mag}$ (Steele \& Jameson 1995; Martín et al. 1996). Given the proximity in wavelength of the $I$ and $Z$ filters it is expected that this dispersion becomes smaller and therefore, the adopted shift takes into account possible binarity effects. For example, PPl 15 was first claimed to be a photometric binary in Paper II and actually it has been confirmed as a double-lined spectroscopic binary with nearly identical components (Basri \& Martín 1998). Those objects fainter than HHJ 3 and PPl 15 and located on the right side of the straight line are considered our best BD candidates. There are $43 \mathrm{BD}$ candidate members of the Pleiades in total, plus one (slightly brighter) very lowmass candidate stellar member of the cluster. A better definition of the true location of this line should be derived after IR photometry and spectroscopy are obtained for the candidates (Zapatero Osorio et al. 1998b).

In Table 3 we list the names, magnitudes, colours and positions for the proposed Pleiades BD candidates. They are named after the Roque Observatory followed by the word Pleiades and numbered according to their decreasing $I$-band apparent magnitude (second column of Table 3). Hereafter, we will use an abridged version of the names which omits the term "Pleiades". The names of the candidates adopting the IAU rules are also pro-

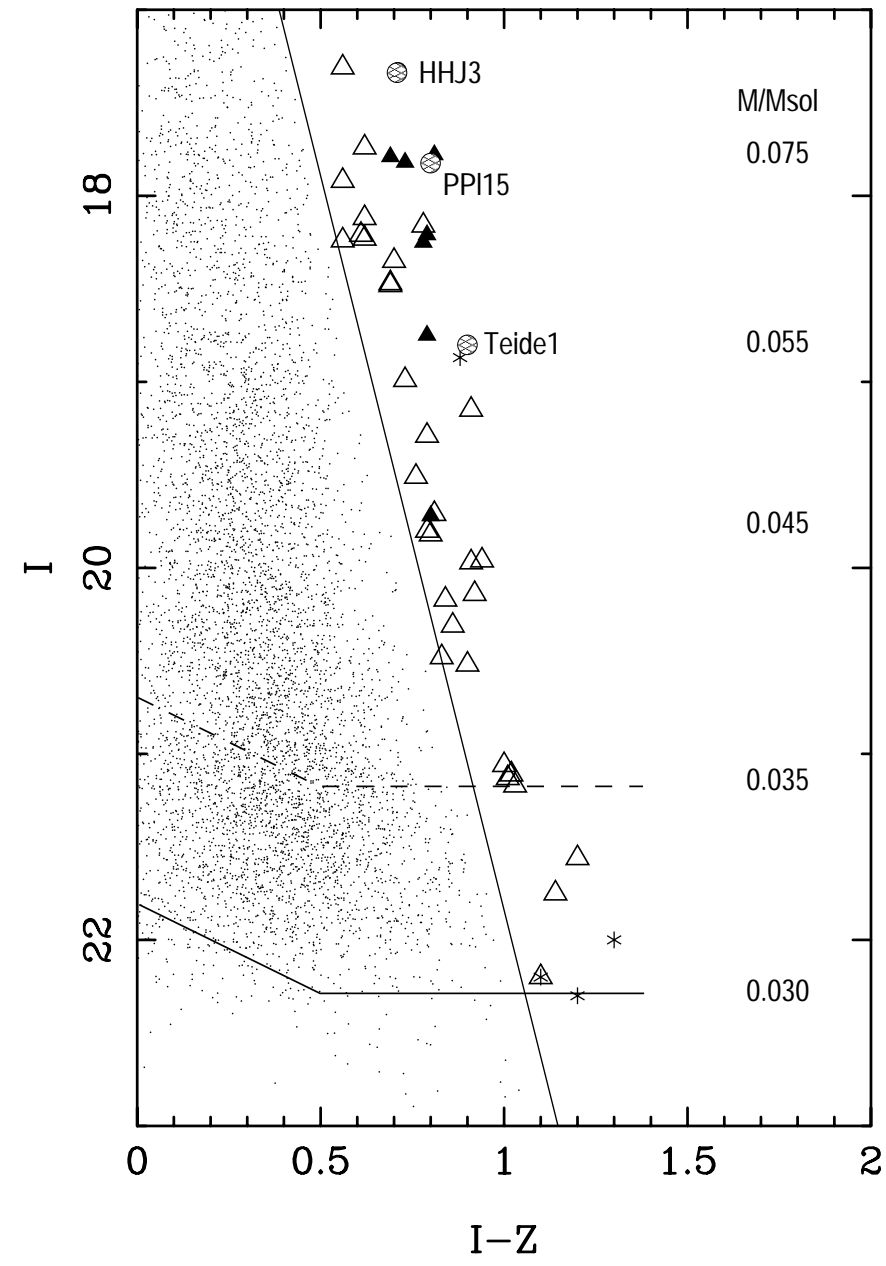

Fig. 3. $I Z$ colour-magnitude diagram for our $1.05 \mathrm{deg}^{2}$ survey in the Pleiades. $Z$ magnitudes are not on a standard system (see text for details). Previous known members are labelled along with the completeness (dashed line) and limiting (full horizontal line) magnitudes. Suspected extended objects are shown with asterisks, and the seven candidates previously studied in Zapatero Osorio et al. (1997a) are indicated with filled triangles. Masses according to the NG Chabrier et al.'s (1996) model for solar metallicity and $120 \mathrm{Myr}$ are labelled on the right side

vided (first column), where the acronym "RPL" stands for Roque Pleiades. Three of the four faintest candidates have slightly larger fwhm than the average value for our frames. Presumably this is an indication that they are not a point source. It is expected that distant galaxies fainter than $I=21$ will begin to contaminate the number counts of objects. Those candidates labelled as extended are shown with a different symbol in Fig. 3. By reference to the reddening map provided in Breger (1987), Roque 3, 5, 15, 18 and 32 could suffer from a somewhat enhanced extinction as they lay within or very near to the CO contours given by the author.

In addition to the INT data, we have obtained $R$-band photometry for five of the candidates at the $2.5 \mathrm{~m}$ Nordic 
Table 3. Coordinates and photometry for the candidates

\begin{tabular}{|c|c|c|c|c|c|c|c|c|}
\hline IAU Name ${ }^{a}$ & $\begin{array}{c}\text { Abridged } \\
\text { name }\end{array}$ & $\begin{array}{l}\text { RA (J20 } \\
\left(\begin{array}{lll}h & \mathrm{~m} & \mathrm{~s}\end{array}\right) \\
\end{array}$ & $\begin{array}{l}\text { 0) DEC } \\
\left(\begin{array}{cc}0 & \prime\end{array}{ }^{\prime \prime}\right) \\
\end{array}$ & Epoch & $I$ & $I-Z$ & $R-I$ & Other names ${ }^{b}$ \\
\hline \multirow[t]{2}{*}{ RPL J034741+2244.5 } & Roque 48 & 34741.3 & 224433 & 1996.123 & 17.31 & 0.56 & 1.73 & \\
\hline & HHJ $3^{\mathrm{c}}$ & 34850.4 & 224430 & 1989.9 & 17.33 & 0.71 & 2.21 & \\
\hline RPL J034904+2333.7 & Roque 47 & 34904.8 & 233340 & 1996.726 & 17.74 & 0.62 & & \\
\hline RPL J034723+2242.6 & Roque 17 & 34723.9 & 224238 & 1996.123 & 17.78 & 0.81 & 2.31 & \\
\hline RPL J034739+2436.4 & Roque 16 & 34739.0 & 243622 & 1996.112 & 17.79 & 0.69 & 2.24 & CFHT-PL-11 \\
\hline \multirow[t]{2}{*}{ RPL J034541+2354.2 } & Roque 15 & 34541.2 & 235410 & 1996.723 & 17.82 & 0.73 & & $\mathrm{PPl} 1$ \\
\hline & $\mathrm{PPl} 15^{\mathrm{c}}$ & $\begin{array}{lll}348 & 04.8\end{array}$ & 233932 & & 17.82 & 0.80 & 2.28 & \\
\hline RPL J034953+2359.0 & Roque 46 & 34953.7 & 235901 & 1996.726 & 17.92 & 0.56 & & \\
\hline RPL J034738+2238.7 & Roque 44 & 34738.7 & 223841 & 1996.123 & 18.12 & 0.62 & 2.01 & \\
\hline RPL J034813+2428.1 & Roque 43 & 34813.8 & 242803 & 1996.726 & 18.16 & 0.78 & & JS $1, \mathrm{PPl} 3$ \\
\hline RPL J034643+2424.9 & Roque 14 & 34643.0 & 242451 & 1996.723 & 18.21 & 0.79 & & \\
\hline RPL J034939+2334.9 & Roque 42 & 34939.3 & 233455 & 1996.726 & 18.21 & 0.61 & & \\
\hline RPL J034644+2435.0 & Roque 41 & 34644.4 & 243500 & 1996.726 & 18.23 & 0.62 & & \\
\hline RPL J035000+2428.3 & Roque 40 & 35000.3 & 242816 & 1996.723 & 18.24 & 0.56 & & \\
\hline RPL J034550+2409.1 & Roque 13 & 34550.6 & 240903 & 1996.723 & 18.25 & 0.78 & 2.32 & \\
\hline RPL J034637+2435.0 & Roque 38 & 34637.7 & 243502 & 1996.726 & 18.35 & 0.70 & & \\
\hline RPL J034819+2425.2 & Roque 12 & 34819.0 & 242512 & 1996.726 & 18.47 & 0.69 & & NPL 36 \\
\hline RPL J034844+2421.3 & Roque 37 & 34844.1 & 242118 & 1996.726 & 18.48 & 0.69 & & \\
\hline RPL J034712+2428.5 & Roque 11 & 34712.0 & 242831 & 1996.723 & 18.75 & 0.79 & 2.68 & NPL 37 \\
\hline TPL J034718+2422.5 & Teide $1^{\mathrm{c}}$ & 34718.0 & 242231 & 1994.85 & 18.80 & 0.90 & 2.74 & \\
\hline RPL J034802+2400.1 & Roque $10^{\mathrm{d}}$ & 34802.1 & 240003 & 1996.723 & 18.87 & 0.88 & & \\
\hline RPL J034623+2420.6 & Roque 9 & 34623.2 & 242037 & 1996.723 & 18.99 & 0.73 & & \\
\hline RPL J034921+2334.0 & Roque 8 & 34921.1 & 233402 & 1996.726 & 19.15 & 0.91 & & \\
\hline RPL J034340+2430.2 & Roque 7 & 34340.3 & 243011 & 1996.726 & 19.29 & 0.79 & & CFHT-PL-24 \\
\hline RPL J034957+2341.8 & Roque 6 & 34957.8 & 234150 & 1996.726 & 19.51 & 0.76 & & \\
\hline RPL J034422+2339.0 & Roque 5 & 34422.4 & 233901 & 1996.726 & 19.71 & 0.81 & & \\
\hline RPL J034353+2431.2 & Roque 4 & 34353.5 & 243111 & 1996.726 & 19.72 & 0.80 & 2.30 & \\
\hline RPL J034410+2340.3 & Roque 3 & 34410.9 & 234015 & 1996.726 & 19.80 & 0.79 & & \\
\hline RPL J034420+2439.0 & Roque 36 & 34420.8 & 243902 & 1996.723 & 19.82 & 0.80 & & \\
\hline RPL J034737+2429.0 & Roque 34 & 34737.5 & 242859 & 1996.723 & 19.96 & 0.94 & & \\
\hline RPL J034849+2420.5 & Roque 33 & 34849.0 & 242025 & 1996.726 & 19.97 & 0.91 & & NPL 40 \\
\hline RPL J034705+2324.9 & Roque 32 & 34705.8 & 232452 & 1996.726 & 20.14 & 0.92 & & \\
\hline RPL J035032+2408.9 & Roque 31 & 35032.5 & 240853 & 1996.726 & 20.17 & 0.84 & & \\
\hline RPL J035016+2408.5 & Roque 30 & 35016.0 & 240835 & 1996.726 & 20.31 & 0.86 & & \\
\hline RPL J035005+2342.2 & Roque 29 & 35005.9 & 234214 & 1996.726 & 20.48 & 0.83 & & \\
\hline RPL J035020+2408.7 & Roque 28 & 35020.7 & 240841 & 1996.726 & 20.52 & 0.90 & & \\
\hline RPL J034746+2403.7 & Roque 27 & 34746.9 & 240342 & 1996.726 & 21.06 & 1.00 & & \\
\hline RPL J034412+2343.3 & Roque 18 & 34412.6 & 234317 & 1996.726 & 21.11 & 1.02 & & \\
\hline RPL J034849+2245.9 & Roque 26 & 34849.3 & 224551 & 1996.123 & 21.13 & 1.01 & 1.98 & \\
\hline RPL J034830+2244.9 & Roque 25 & 34830.6 & 224450 & 1996.123 & 21.17 & 1.03 & 2.75 & \\
\hline RPL J034951+2515.5 & Roque 2 & 34951.4 & 251531 & 1996.117 & 21.31 & & $\geq 2.1$ & \\
\hline RPL J034321+2434.7 & Roque 24 & 34321.4 & 243442 & 1996.726 & 21.56 & 1.20 & & \\
\hline RPL J034751+2355.8 & Roque 23 & 34751.0 & 235548 & 1996.726 & 21.75 & 1.14 & & \\
\hline RPL J034321+2432.0 & Roque $22^{\mathrm{d}}$ & 34321.3 & 243202 & 1996.726 & $22.0:$ & 1.3: & & \\
\hline RPL J034327+2433.7 & Roque $21^{\mathrm{d}}$ & 34327.8 & 243340 & 1996.726 & $22.2:$ & 1.1: & & \\
\hline RPL J034843+2240.8 & Roque 20 & 34843.7 & 224046 & 1996.123 & $22.2:$ & 1.1: & & \\
\hline RPL J034855+2420.2 & Roque $19^{\mathrm{d}}$ & 34855.3 & 242009 & 1996.726 & 22.3: & 1.2: & & \\
\hline
\end{tabular}

a "RPL" stands for Roque Pleiades, and "TPL" for Teide Pleiades.

b References: PPl objects from Stauffer et al. (1989); JS objects from Jameson \& Skillen (1989); NPL objects from Festin (1998); CFHT-PL objects from Bouvier et al. (1998).

c Coordinates for HHJ 3 taken from Hambly et al. (1993); for PPl 15 from Stauffer et al. (1994); coordinates and $R I$ photometry for Teide 1 taken from Paper I.

d These objects appear slightly extended in the $I Z$ images.

: For error bars in the photometry see text. Those measurements labelled with a ":" have rather large uncertainties. 
Optical Telescope (BroCam1, NOT) on 1996 October 1011 (Roque 17, 11 and 4), and at the $1 \mathrm{~m}$ Jacobus Kaptein Telescope (JKT) on 1996 September 12-13 (Roque 16 and 13), both telescopes at the ORM. The CCDs used were a Tektronix $1024 \times 1024$ providing fields of view of 3.0 and $5.5 \operatorname{arcmin}^{2}$, respectively. Exposure times were typically $15 \mathrm{~min}$ at NOT and $30 \mathrm{~min}$ at JKT. Landolt's (1992) standard stars were observed just before and after the targets. Reduction of the raw frames and photometry of the candidates has been performed as described above. Uncertainties in $R$ magnitudes range from $0.07 \mathrm{mag}$ for the brightest objects to $0.15 \mathrm{mag}$ for the faintest ones. Considering the $R-I$ photometry from Table 3 and from other deep surveys (Paper I; Bouvier et al. 1998) Roque 44 and Roque 26 are not likely to be Pleiades members as they seem to deviate towards bluer colours from the sequence defined by other candidates.

Astrometry for all Roque objects has been performed by the triangles fitting method using the APM Sky Catalogue. Several stars close to every candidate were identified and they served as a reference for the astrometric calibration. Coordinates are accurate to approximately \pm 2 . The location of our candidates in the surveyed area is depicted in Fig. 4. Their distribution around the cluster center appears quite homogeneous. However, we note that the number of fields (9) with $\geq 3$ BD candidates is surprisingly large compared to the expectations from a random distribution. The study of possible spatial inhomogeneities within the cluster still awaits membership confirmation. Seven of the Roque BD candidates have also been identified in other surveys. The last column of Table 3 gives cross-identifications. Our $I$ magnitudes seem to be on average $0.25 \mathrm{mag}$ brighter than those available in the most recent literature. This is likely due to an effect of the colour-dependence of the Harris filters we used in our observations; although a red standard star was considered, it is poorly calibrated and consequently does not provide an accurate determination of the colour-term in the photometric calibration. Cossburn et al. (1998) have found that the colour-term for the transformation from Harris $I$ to Cousins is indeed rather significant for very red objects. In the case of Roque 33 (NPL 40) the difference found is -0.58 mag which might be due to contamination from a nearby very bright star (and saturated in our frames). Figure 5 provides the $I$-band finder charts $\left(2^{\prime} \times 2^{\prime}\right.$ in extent) for all Roque objects ordered as listed in Table 3.

According to the "NextGen" (NG) theoretical evolutionary models of Chabrier et al. (1996), and adopting solar metallicity, an age of $120 \mathrm{Myr}$ (Basri et al. 1996; Martín et al. 1998; Stauffer et al. 1998) and a distance of $127 \mathrm{pc}$ for the Pleiades cluster, our survey has detected objects in the mass interval from roughly $0.08 M_{\odot}$ down to $0.03 M_{\odot}$. The completeness magnitudes correspond to $0.035 M_{\odot}$ as indicated in Fig. 3. Chabrier et al.'s models provide absolute magnitudes as a function of mass, metallicity and age obtained by direct integration of the-

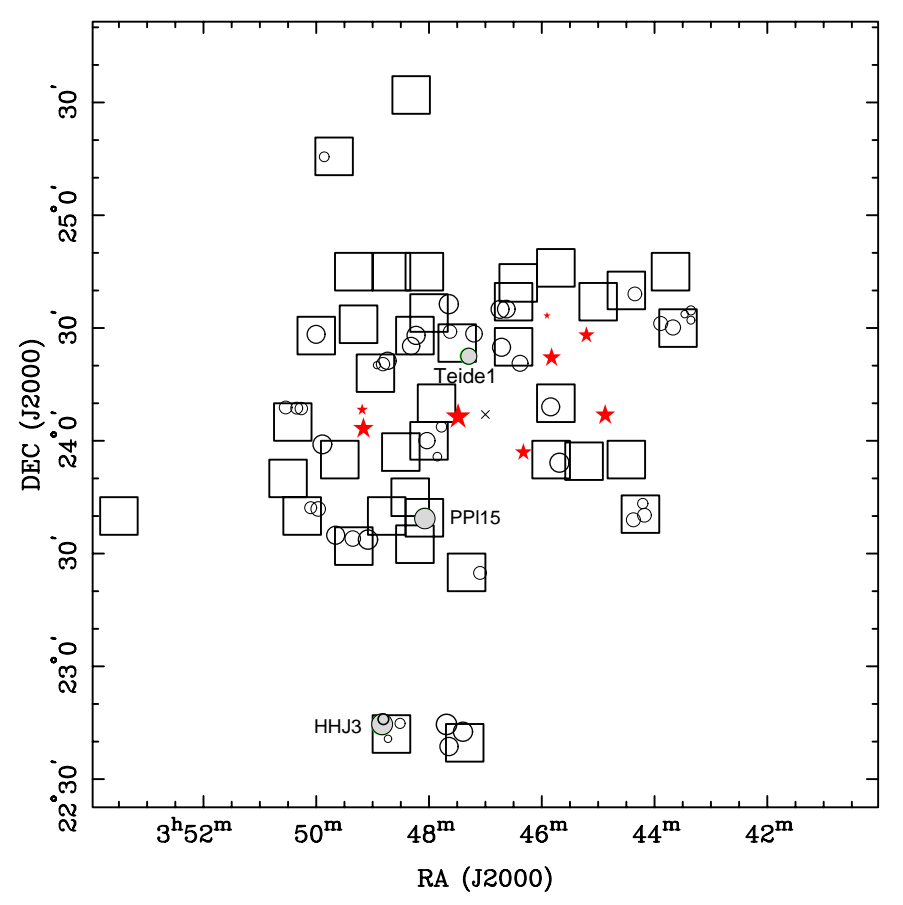

Fig. 4. Location of our candidates within the fields observed in our survey covering $1.05 \mathrm{deg}^{2}$. As in Fig. 1 central coordinates $\left(3^{\mathrm{h}} 47^{\mathrm{m}},+24^{\circ} 7^{\prime}\right.$, Eq. 2000) are indicated with a cross. Filled star symbols outline stars brighter than $6^{\text {th }}$ magnitude. The relative brightness is represented by symbol diameters. North is up and East is left

oretical atmospheres which do not incorporate grain formation and dust absorption (Allard et al. 1997). However, the effects of condensation become important for temperatures cooler than about $2500 \mathrm{~K}$ (Tsuji et al. 1996; Jones \& Tsuji 1997), a temperature range partially covered by our survey. Preliminary computations by Baraffe (private communication) show that models considering dust formation and opacities predict brighter $I$ magnitudes and subsequently slightly lowers the mass determination by $\sim 8 \%$.

Membership and therefore the real nature of our candidates on the basis of $J H K$ photometry and spectroscopy and the Pleiades mass function will be addressed in a forthcoming paper (Zapatero Osorio et al. 1998b). Seven of them (Roque 17, 16, 15, 14, 13, 11 and 4) with $I$ magnitudes in the range $17.8-19.5$ (masses in the interval $\left.0.08-0.045 M_{\odot}\right)$ have already been studied to some extent by Zapatero Osorio et al. (1997a). They are shown in Fig. 3 with a different symbol. The authors conclude that given their $K$ magnitudes, radial velocities, spectral types and weakness of some atomic features these candidates should be considered as Pleiades members. The number of remaining candidates in our $I Z$ survey deserve further investigation as there are large enough to ensure that follow-up observations will confirm more Pleiades substellar objects. Among the faintest ones, there could be BDs with masses as low as $0.03 M_{\odot}$. These studies will make it 
possible to derive the cluster mass function well into the substellar regime.

\section{Conclusions}

We have imaged about $1 \mathrm{deg}^{2}$ in the central region of the Pleiades young cluster using $I Z$ broad-band filters, and identified more than 40 brown dwarf candidates with $I$ magnitudes ranging from 17.5 to 22 (completeness is given by $I \sim 21 \mathrm{mag}$ ). This corresponds to a mass interval starting roughly at the substellar mass limit down to $0.03 M_{\odot}$ according to recent non-dusty evolutionary models by Chabrier et al. (1996). Follow-up IR and spectroscopic observations of seven of these candidates (Zapatero Osorio et al. 1997a) have revealed that they are very likely to be cluster brown dwarfs. Further data for the remaining candidates should definitely establish or refute cluster membership in the Pleiades. These studies will provide a solid foundation for deriving the cluster substellar mass function.

Acknowledgements. We thank the referee, J. Stauffer, for his valuable comments. We also thank the Comité Científico Internacional for awarding $25 \%$ of the International Time at the Observatories of the Canary Islands to this project in 1996-97. Partial financial support was provided by the Spanish DGES project no. PB95-1132-C02-01. A. M. acknowledges a NATO-CNR senior fellowship.

\section{References}

Allard F., Hauschildt P.H., Alexander D.R., Starrfield S., 1997, ARA\&A 35, 137

Basri G., Marcy G.W., Graham J.R., 1996, ApJ 458, 600

Basri G., Martín E.L., 1998, in: Brown Dwarfs and Extrasolar Planets, Rebolo R., Martín E.L., Zapatero Osorio M.R. (eds.), ASP 134, 284

Bessell M.S., 1991, AJ 101, 662
Bouvier J., Stauffer J.R., Martín E.L., Barrado y Navascués D., Wallace B., Béjar V.J.S., 1998, A\&A 336, 490

Breger M., 1987, ApJ 319, 754

Cossburn M.R., Hodgkin S.T.H., Jameson R.F., 1998, MNRAS (submitted)

Chabrier G., Baraffe I., Plez B., 1996, ApJ 459, L916

Cossburn M.R., Hodgkin S.T., Jameson R.F., Pinfield D.J., 1997, MNRAS 288, L23

Festin L., 1998, A\&A 333, 497

Hambly N.C., 1998, in: Brown Dwarfs and Extrasolar Planets, Rebolo R., Martín E.L., Zapatero Osorio M.R. (eds.), ASP 134, 11

Hambly N.C., Hawkins M.R.S., Jameson R.F., 1993, A\&AS 100,607

Jameson R.F., Skillen I., 1989, MNRAS 239, 247

Jones H.R.A., Tsuji T., 1997, ApJ 480, L39

Landolt A.U., 1992, AJ 104, 340

Martín E.L., Basri G., Gallegos J.E., et al., 1998, ApJ 499, L61

Martín E.L., Rebolo R., Zapatero Osorio M.R., 1996, ApJ 469, 706

Rebolo R., Martín E.L., Basri G., et al., 1996, ApJ 469, L53

Rebolo R., Zapatero Osorio M.R., Martín E.L., 1995, Nat 377, 129

Stauffer J.R., Hamilton D., Probst R.G., Rieke G., Mateo M., 1989, ApJ 344, L21

Stauffer J.R., Hamilton D., Probst R.G., 1994, AJ 108, 155

Stauffer J.R., Hartmann L., 1987, ApJ 318, 337

Stauffer J.R., Schultz G., Kirkpatrick J.D., 1998, ApJ 499, 199

Steele I., Jameson R.F., 1995, MNRAS 272, 630

Tsuji T., Ohnaka K., Aoki W., 1996, ApJ 305, L1

van Leeuwen F., 1983, PhD thesis, Univ. Leiden

Zapatero Osorio M.R., Rebolo R., Martín E.L., 1997b, A\&A 317, 164 (Paper I)

Zapatero Osorio M.R., Martín E.L., Rebolo R., 1997c, A\&A 323, 105 (Paper II)

Zapatero Osorio M.R., Rebolo R., Martín E.L., et al., 1997a, ApJ 491, L81

Zapatero Osorio M.R., Rebolo R., Martín E.L., et al., 1998a, in: Brown Dwarfs and Extrasolar Planets, Rebolo R., Martín E.L., Zapatero Osorio M.R. (eds.), ASP 134, 51

Zapatero Osorio M.R., et al., 1998b, A\&A (in preparation) 